\title{
Targeting RNA helicase DDX3 in stem cell maintenance and teratoma formation
}

\author{
Candace L. Kerr ${ }^{1}$, Guus M. Bol2,3 Farhad Vesuna ${ }^{2}$ and Venu Raman 2,4,5 \\ ${ }^{1}$ Department of Gynecology and Obstetrics, Johns Hopkins University School of Medicine, Baltimore, MD, USA \\ ${ }^{2}$ Department of Radiology and Radiological Science, Johns Hopkins University School of Medicine, Baltimore, MD, USA \\ ${ }^{3}$ Department of Oncology, University Medical Center Utrecht Cancer Center, GA Utrecht, The Netherlands \\ ${ }^{4}$ Department of Oncology, Johns Hopkins University School of Medicine, Baltimore, MD, USA \\ ${ }^{5}$ Department of Pathology, University Medical Center Utrecht Cancer Center, GA Utrecht, The Netherlands \\ Correspondence to: Venu Raman, email: vraman2@jhmi.edu
}

Keywords: DDX3; stem cells; differentiation; RK-33; teratoma

Received: November 21, $2018 \quad$ Accepted: January 13,2019 Published: January 25, 2019

Copyright: Kerr et al. This is an open-access article distributed under the terms of the Creative Commons Attribution License 3.0 (CC BY 3.0), which permits unrestricted use, distribution, and reproduction in any medium, provided the original author and source are credited.

\section{ABSTRACT}

DDX3 is an RNA helicase that has antiapoptotic properties, and promotes proliferation and transformation. Besides the role of DDX3 in transformed cells, there is evidence to indicate that DDX3 expression is at its highest levels during early embryonic development and is also expressed in germ cells of adults. Even though there is a distinct pattern of DDX3 expression during embryonic development and in adults, very little is known regarding its role in embryonic stem cells and pluripotency. In this work, we examined the relationship between DDX3 and human embryonic stem cells and its differentiated lineages. DDX3 expression was analyzed by immunohistochemistry in human embryonic stem cells and embryonal carcinoma cells. From the data obtained, it was evident that DDX3 was overexpressed in undifferentiated stem cells compared to differentiated cells. Moreover, when DDX3 expression was abrogated in multiple stem cells, proliferation was decreased, but differentiation was facilitated. Importantly, this resulted in reduced potency to induce teratoma formation. Taken together, these findings indicate a distinct role for DDX3 in stem cell maintenance.

\section{INTRODUCTION}

Despite the enormous potential of pluripotent stem cells for the treatment of human disease, a significant gap exists with respect to our understanding of the biological controls that regulate their differentiation and pluripotent nature. Thus, defining molecular markers that are essential for the maintenance of the undifferentiated state of these cells will help us understand how to drive their differentiation into more specialized cells. The discovery of factors that regulate stem cell maintenance began with the characterization of transcription factors that were required for stem cell self-renewal and pluripotency. These factors include Oct4 (also known as POU5F1 (POU domain, class 5, transcription factor 1), SRY (sex determining region Y)-box 2 (Sox2), and Nanog as well as others, which are master regulators of stem cell fate
[1-4]. Since their discoveries it has been realized that mechanisms must be in place for a stem cell to maintain its pluripotent state. These mechanisms have included key players in epigenetic regulation, cell cycle, and autophagy [5-12].

Even though significant advancement has been made to understand the mechanics of stem cell maintenance and differentiation, there are still many regulatory pathways, that are not clearly understood. In our quest to categorize biomarkers for stem cell characteristics, we identified DEAD-Box Helicase 3 (DDX3) as a potential gene candidate. DDX3, also known as DDX3X because of its location on the $\mathrm{X}$ chromosome, is a member of the DEAD-box RNA helicase family which is involved in transcription, RNA splicing, nuclear export of mRNA, and translation initiation [13-16]. DDX proteins are expressed in a wide variety of cell types and are highly expressed 
during embryogenesis and in diverse human cancers. The precise roles of DDX3 in development and cancer are unclear $[17,18]$. Evidence in lower organisms has indicated roles of DDX3 in early stem cell proliferation of the embryo and in mammals, regulating apoptosis and the cell cycle. In humans, the sex-specific DEAD box gene, DDX3X (also known as DBX and CAP-Rf), resides at Xp11.3-p11.23 and does not undergo X-inactivation in females $[19,20]$. It has a functional homolog on the Y chromosome, DBY (91.7\% homology), and this gene product has an activity that is crucial for proliferation in the spermatogonia of mice with inferred evidence in azoospermic men [21-26].

Although, a role for DDX3 has not been reported in human stem cells, its role in stem cell maintenance and differentiation is beginning to be unraveled in lower organisms. For example, the homolog of DDX3 in the planarian flatworm, SpolvlgA, has been demonstrated to be expressed from the first cleavage rounds in blastomere cells and blastomere-derived embryonic cells [27]. These cells are undifferentiated and they engage in a massive wave of differentiation during subsequent stage development. Interestingly, in adult worms, SpolvlgA is expressed in spermatogonia, spermatocytes and differentiating spermatids. Similarly in other invertebrates, the DDX3 homologue in Botryllus schlosseri (Urochordata), BS-PL10 modulates this animal's blastogenic cycle, increasing from blastogenic stage A to blastogenic stage D [28] while a sharp decrease in BSPL10 expression occurs during organogenesis such that the highest levels of expression is observed in multipotent soma and germ cells. Also, Ddx3x heterozygous female mice exhibits placental abnormalities during development and is embryonic lethal [29]. In addition, loss of ddx $3 x$ results in widespread apoptosis due to enhanced DNA damage and cell cycle arrest [29]. Thus, together with the evolutionary conservation of DDX3 [30], evidence points to this as an ancestral gene with defined functional roles both in self-renewal and pluripotency.

Here, we report that DDX3 promotes stem cell maintenance. Specifically, we show that undifferentiated embryonic stem cells (ESC) and embryonal carcinoma cells (ECCs) express high levels of DDX3 compared to differentiated cells. Notably, when DDX3 activities were perturbed, we observed a drastic decrease in the proliferation of undifferentiated stem cells along with an increase in cellular differentiation. Moreover, we also confirmed in vivo that inhibiting DDX3 activity prevents teratoma formation in NOD-scidIL-2R $\gamma$ null (NOG) mice. Taken together, our results indicate that DDX3 is an integral component of stem cell character and regulating DDX3 activity could be used to control differentiation and pluripotency.

\section{RESULTS}

\section{DDX3 expression decreases with differentiation in human ESCs and ECCs}

Following gene expression analysis of pluripotent ESCs and unipotent progenitors of embryonic germ cells (EGCs) and ECCs known as primordial germ cells (PGCs), DDX3 was identified as one of a few genes that showed differential expression between these two cell types. To confirm this finding, qRT-PCR analysis was performed, which showed that DDX3 mRNA expression is significantly higher in ESCs and ECCs than in their differentiated counterparts of neural lineage (NRN) and human fetal fibroblasts (hFF) compared to primordial germ cells (baseline), which are the unipotent, or more differentiated progenitors of EGCs and ECCs (Figure 1). This was further corroborated by using three independent DDX3 specific primer sets (data not shown). Importantly, evidence comparing EGC to the PGC from which they are

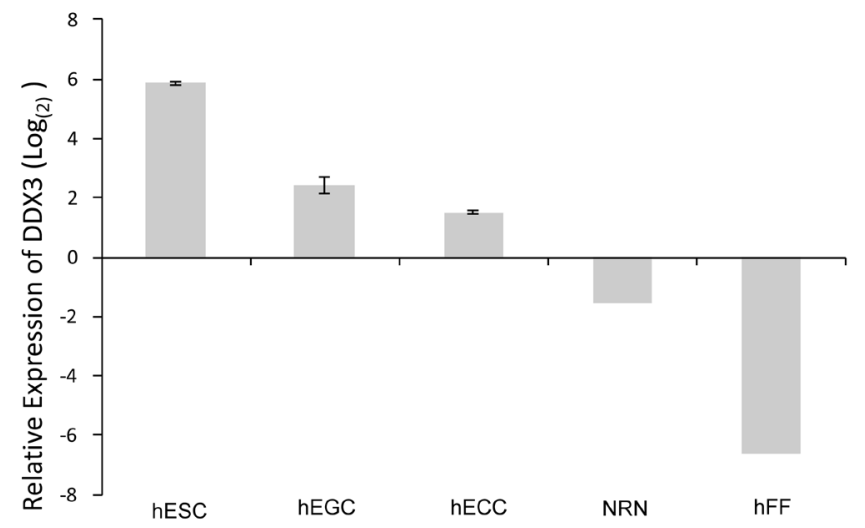

Figure 1: Expression of DDX3 in pluripotent and differentiated cell lines. DDX3 expression is lower in differentiated cells (FF: human fetal fibroblasts; ECC Neuro: Neural differentiated hECCs) and higher in pluripotent stem cells (hEGCs, hECCs and hESCs). Relative expression of $D D X 3$ was compared to $\beta$-actin as the endogenous control. $\Delta \Delta \mathrm{Ct}$ method was also employed using the unipotent germ cell progenitor cells, PGCs as the baseline value $(N=3, P<0.05)$. 
derived indicates that DDX3 may be involved in the initial stages driving pluripotency.

\section{Altered DDX3 expression levels following differentiation of ESCs and ECCs}

As DDX3 levels were altered following differentiation, we analyzed DDX3 expression by immunofluorescence to determine the expression pattern at the cellular level. As show in Figure 2, DDX3 expression was significantly reduced after differentiation of ECCs demonstrating that undifferentiated ECCs that express OCT4 (Figure 2A) also express DDX3 (Figure 2B). More importantly, when cultured under neural-inducing conditions DDX3 expression is ablated (Figure 2E). This is evident by the lack of DDX3 expression in cells (Figure 2E) which have little or no expression of the pluripotent cell surface marker TRA-1-60 (Figure 2D) compared to the undifferentiated ECCs known to express both TRA-1-60 and OCT4. These results indicate that DDX3 expression is concomitant with pluripotent markers, especially OCT4 and TRA-1-60 expression in ECCs. Similar results were also seen in EGCs and ESCs (data not shown).

\section{Inhibition of DDX3 in undifferentiated hESCs reduces NANOG, OCT4 and SOX2 expression without reducing cell viability}

Next, we carried out immunofluorescence studies on hESCs to measure the levels of protein expression of DDX3, NANOG, OCT4 and SOX2 with and without the DDX3 inhibitor, RK-33. As shown in Figure 3A, DDX3 expression was robust in undifferentiated hESCs along with the expression of NANOG, OCT4 and SOX2. It appeared also that the majority of cells, which were OCT4+ were also DDX3+. However, the addition of 0.1 $\mu \mathrm{M}$ of RK-33, a small molecule inhibitor of DDX3 [3133 ], to these cells significantly decreased DDX3 levels within four days in addition to NANOG, OCT4 and SOX2 suggesting a biological function for DDX3 in maintaining the pluripotent state. Importantly, colony numbers and size was similar with and without RK-33 demonstrating that DDX3 inhibition did not affect cell viability and that RK-33 was non-toxic to the cells at the low concentration tested (Figure 3B and 3C).

\section{DDX3 inhibition promotes hESC differentiation}

As down-regulating DDX3 levels reduced pluripotent markers, we wanted to determine if inhibiting DDX3 promotes differentiating phenotypes. To study this, we compared the ability of hESC to differentiate into neural and mesodermal lineages with and without DDX3 inhibition. This would confirm whether hESC differentiate more efficiently when DDX3 activity was inhibited. For this purpose, hESCs were plated onto matrigel and cultured in media that either induced neural differentiation (NEURO) or mesoderm (MESO) differentiation as previously reported by us and others [34-36]. To this media was added $0.1 \mathrm{uM}$ RK-33 or equivalent DMSO alone as a control and cells examined for differentiation markers after 4 days. At this early time point in differentiation, we would not expect to see a high percentage of cells that have lineage restricted markers. As shown in Figure 4, inhibition of DDX3 by RK-33 $(0.1 \mu \mathrm{M})$ facilitated a higher number of cells with
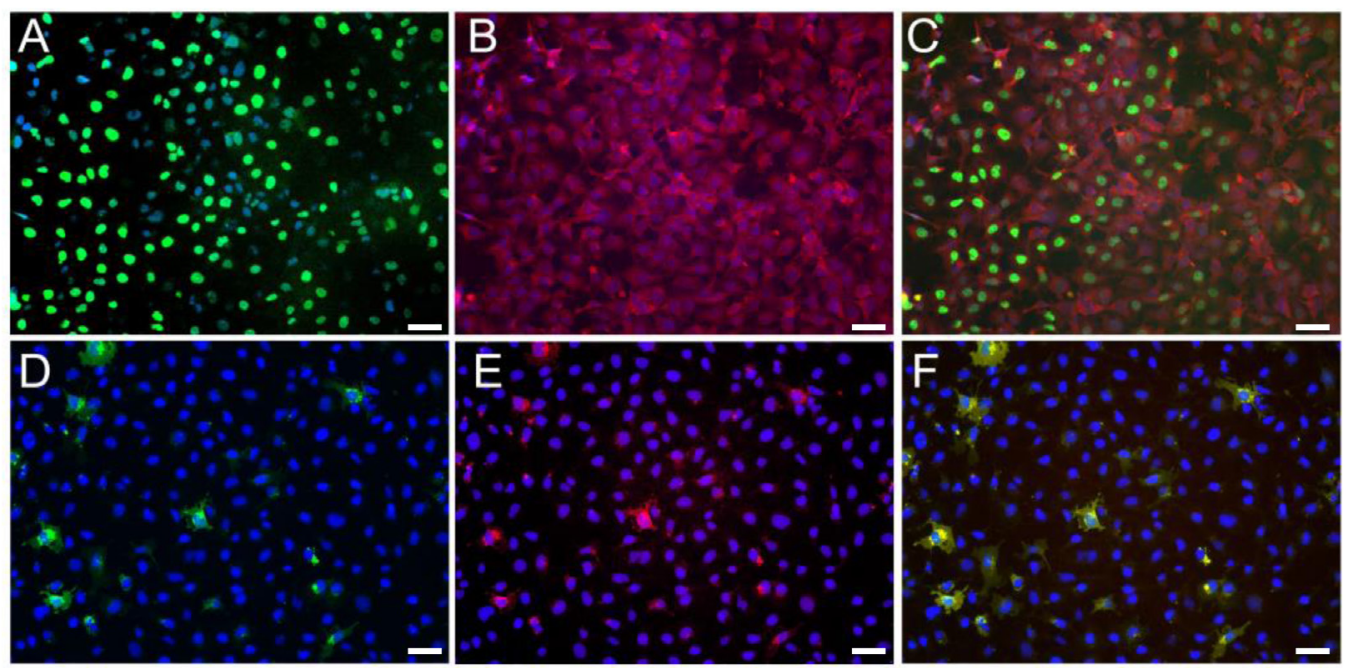

Figure 2: Immunofluorescence detection of DDX3 in undifferentiated human ECCs. A. Oct4 (green), B. DDX3 (red). C. A and B overlaid. Reduced DDX3 in differentiated ECCs showing reduced expression of the pluripotent marker. D. Cell surface expression of Tra-1-60 (green) and E. DDX3 (Red) is reduced in ECCs cultured under neural inducing differentiation. F. Overlay of D and E shows a few remaining undifferentiated ECCs that are TRA-1-60+/DDX3+. DAPI was used as nuclear stain (blue). 
lineage restricted marker expression for both the neural and mesodermal lineage compared to controls (DMSO versus RK-33). Specifically, RK-33 reduced the number of OCT4+, SOX2+ cells and increased the number of cells which expressed neuroectoderm (SSEA1+, NESTIN + ) and mesodermal (Podocalyxin+, CD34+) lineages compared to controls. Together, these results show that DDX3 is an integral effector for maintaining pluripotency.

\section{RK-33 does not inhibit proliferation of human embryonic fibroblast cells at physiological levels}

Given that down regulation of DDX3 by RK33 promotes hESC differentiation, we wanted to determine whether RK-33 inhibits proliferation of the differentiated hESCs at the concentrations we studied as well as determine toxic levels of the drug. To do this we performed a dose response experiment in ESCs exposed to various concentrations of RK-33. As shown in Figure 5A, RK-33 concentration up to $1 \mu \mathrm{M}$ had no effect on hESC proliferation while $5 \mu \mathrm{M}$ and $10 \mu \mathrm{M}$ inhibited hESC proliferation. Moreover at higher levels, $5 \mu \mathrm{M}$ and $10 \mu \mathrm{M}$, differentiated neural cells were most sensitive to RK-33 with higher reductions in living cells $(0 \%$ at $5 \mu \mathrm{M}$ of neural cells compared to mesodermal and undifferentiated cells at $0 \%$ live cells by $25 \mu \mathrm{M}$ RK-33) (Figure 5B). Interestingly, as shown in Figure 5C and 5D, concentrations of RK-33 as used in the following studies had very little effect on the proliferation or survival of human fetal fibroblast cells further suggesting that inhibiting DDX3 is not affecting the proliferation or survival of stem cells. Taken together, these observations help support a role for DDX3 in the differentiation process.

\section{Reduced teratoma formation following RK-33 treatment}

Based on our earlier observation that RK-33 facilitates differentiation and decreases pluripotency markers, we carried out a pilot experiment to determine if

A)
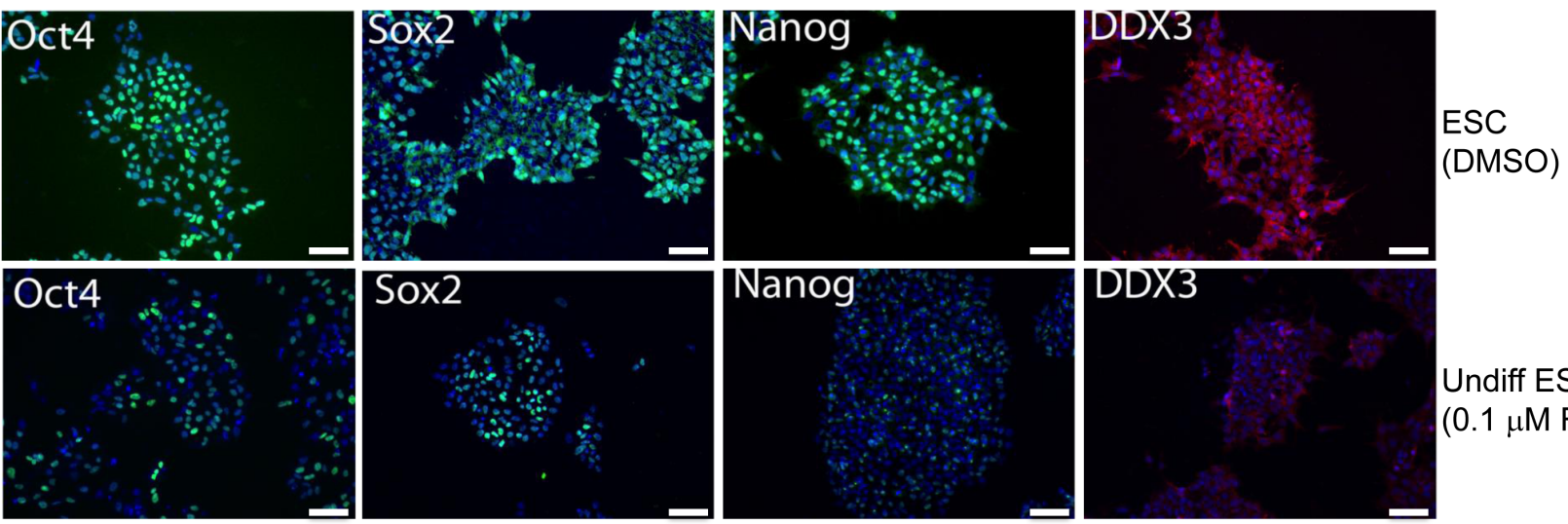

Undiff ESC

(0.1 $\mu \mathrm{M}$ RK-33)

B)
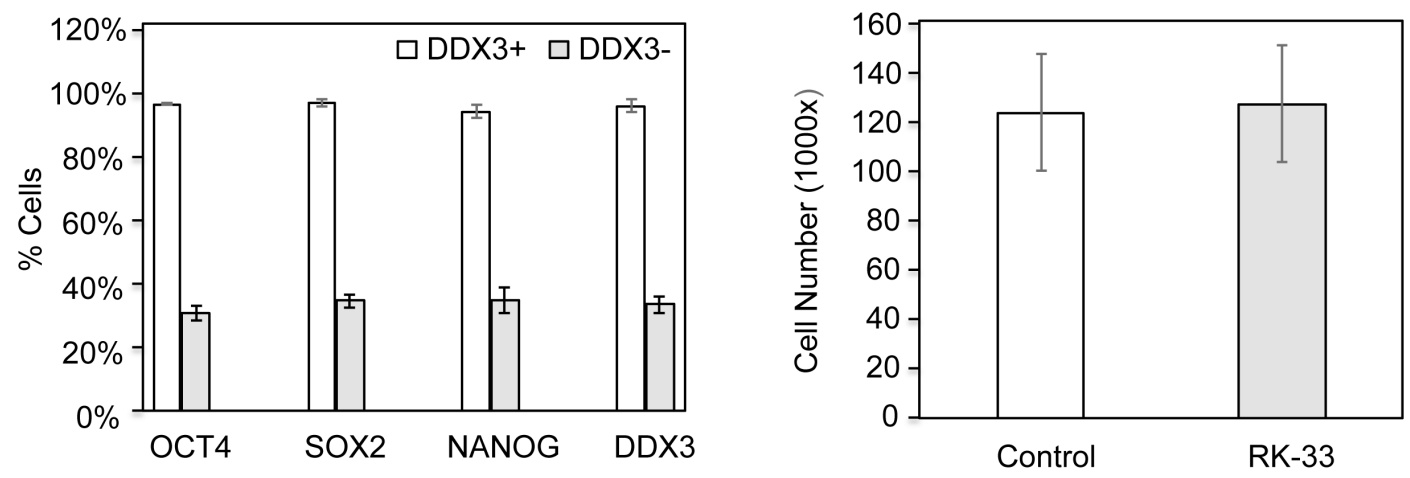

Figure 3: DDX3 expression correlates with pluripotency markers in hESC. A. (Left) hESCs express DDX3 in cells expressing the stem cell markers OCT4, NANOG, and SOX2. (Right) Knock-down of DDX3 activity by RK-33 reduces the expression of NANOG, OCT4 and SOX2 in hESCs. Nucleus was stained with DAPI (Blue). B. Quantitative analysis of the percent of cells, which expressed each marker are presented. While the majority or $>95 \%$ of the undifferentiated hESC expressed all four markers. After inhibition of DDX3 activity, the percent of cells expressing DDX3 was significantly reduced and to a similar extent as the stem cell markers. $(P<0.05)$. C. Bar graph of cell number with DDX3 inhibitor versus control demonstrating survival appeared unaffected by DDX3 inhibition. 
hESCs treated with RK-33 can reduce teratoma formation. As shown in Figure 6, hESCs when injected into the flanks of NOG mice resulted in an average teratoma volume of over $2000 \mathrm{~mm}^{3}$. However, the teratoma volume from hESCs animals treated with RK-33 was barely palpable ( $N=12$ injections with or without RK-33 treatment). This indicates that inhibiting DDX3 activity can enhance differentiation and decrease teratoma growth.

\section{DISCUSSION}

A role for DDX3 in stem cell maintenance and differentiation is beginning to be unraveled in lower organisms. For example, the homologue of DDX3 in the planarian flatworm, SpolvlgA, are expressed from the first cleavage rounds in blastomere cells and blastomere-derived embryonic cells [27]. These cells are undifferentiated and they engage in a massive wave

\section{Meso DMSO only Neuro DMSO only Meso Diff $(0.1 \mu \mathrm{M}) \quad$ Neuro Diff $(0.1 \mu \mathrm{M})$}

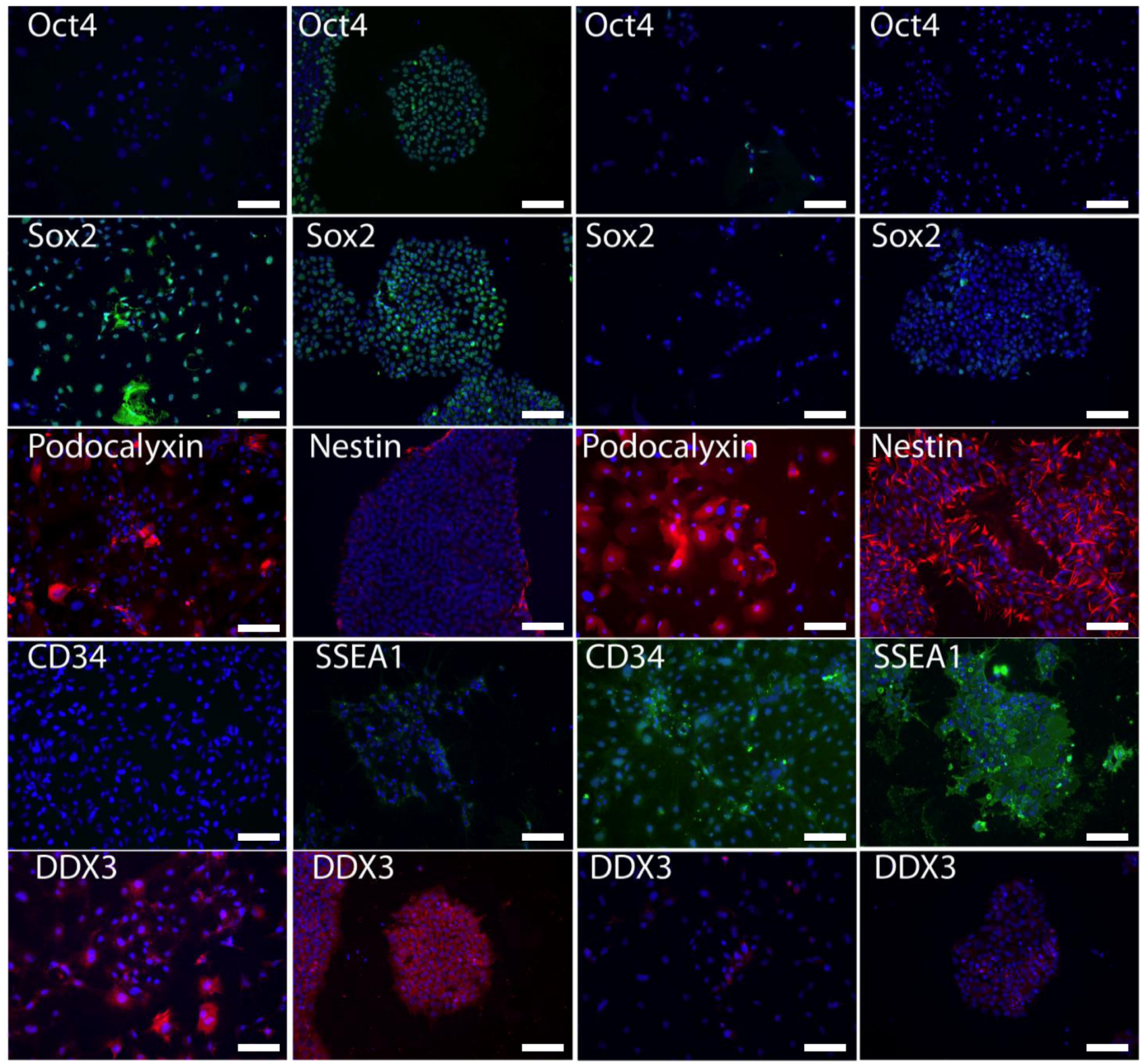

Figure 4: DDX3 inhibition promotes hESC differentiation after 4 days in culture. Left Column vehicle only controls (DMSO). Right Column with 0.1uM RK-33. hESCs exposed to DMSO consisted of more undifferentiated OCT4+, SOX2+ cells and reduced numbers of cells expressing differentiation markers A. mesoderm: Podo, CD34 B. neuroectoderm: Nestin+, SSEA1+ compared to hESC cultured with DDX3 inhibitor RK-33. DAPI (Blue) is nuclear stain. 
of differentiation during subsequent stage development. Interestingly, in adult worms, SpolvlgA is expressed in spermatogonia, spermatocytes and differentiating spermatids. Similarly in other invertebrates, the DDX3 homologue in Botryllus schlosseri (Urochordata), BS-PL10 modulates this animal's blastogenic cycle, increasing from blastogenic stage A to blastogenic stage D [28] while, a sharp decrease in BS-PL10 expression occurred during organogenesis such that the highest levels of expression occurred in multipotent soma and germ cells. Thus, together with the evolutionary conservation of DDX3 [30], evidence points to this as an ancestral gene with defined functional roles both in differentiation and pluripotency. In addition, DDX3 has been associated with the less differentiated phenotypes of some cancers [31-33, 37-39]. For example, we have found that DDX3 activity has been associated with the proliferation and differentiated status of breast cancer cells [38, 40].

We now show that DDX 3 has a role in differentiation by showing its involvement in the pluripotent state of stem

A)

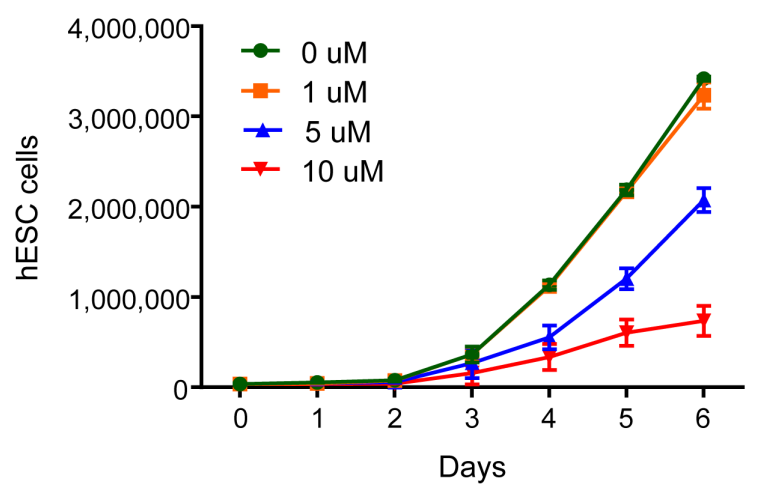

C)

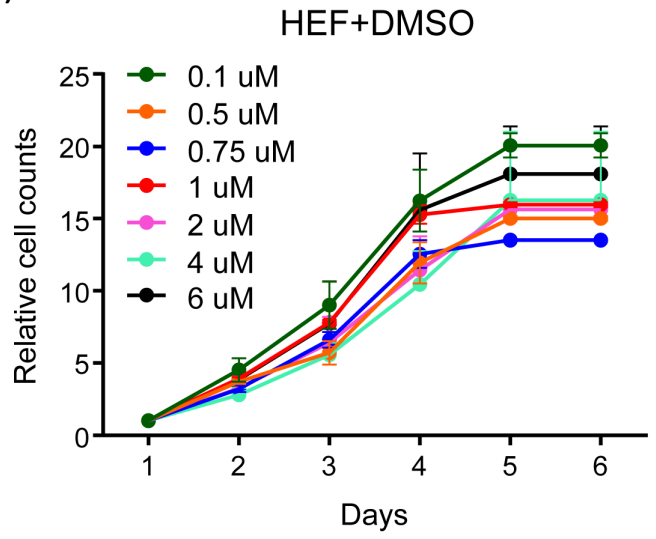

cells. Pluripotent stem cells are defined by their clonal capacity and ability to differentiation into all cell types of the embryo. While they share certain features with cancer cells such as high proliferative capacity and immortality in vitro, they also exhibit a robust differentiation capacity that provides a unique opportunity to study the role of DDX3 in proliferation and their differentiation, which closely mimics development. Using these models, we were able to compare more differentiated cells such as primordial germ cells (PGCs), the progenitor germ cells for sperm and egg, to their undifferentiated pluripotent counterparts, embryonic germ cells (EGCs) and embryonal carcinoma cells (ECCs). EGCs and ECCs are both pluripotent stem cells derived from PGCs, EGCs are derived from PGCs in cell culture while ECCs are cancer stem cells derived from teratocarcinomas organically derived from PGCs in the body. Here, we show that compared to a known differentiated progenitor cell, PGCs DDX3 expression was higher in EGCs and ECCs. DDX3 expression was also higher in ECCs and embryonic stem
B)

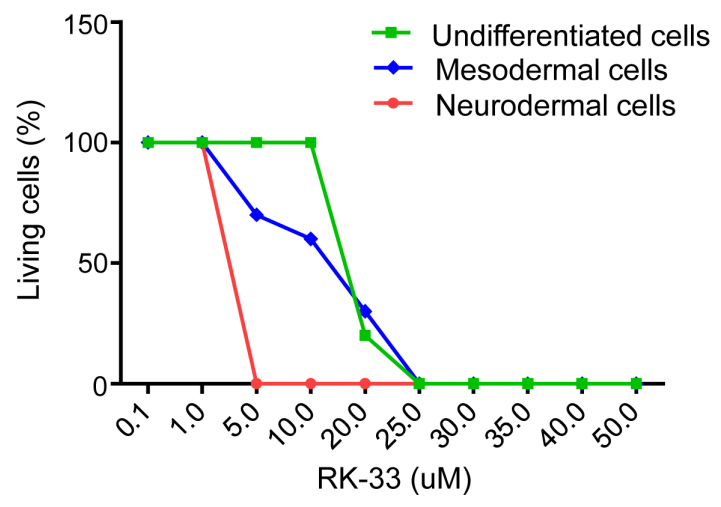

D)

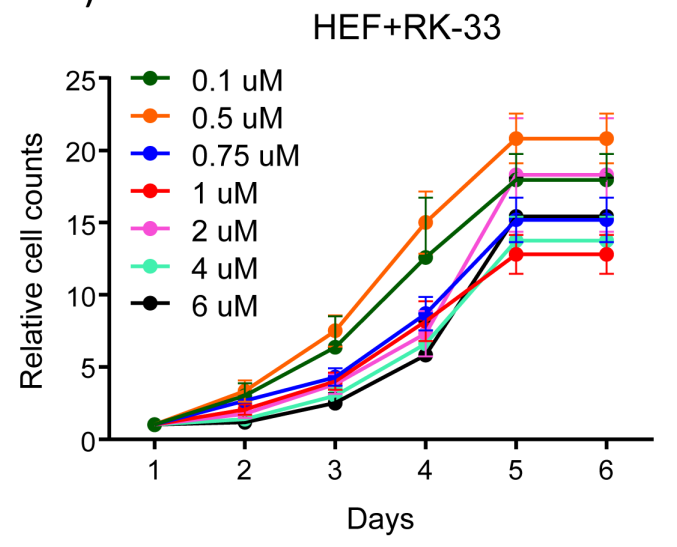

Figure 5: Dose response of the DDX3 inhibitor RK-33 on undifferentiated and differentiated hESCs. A. Proliferation assay using hESCs in the presence of different concentrations of RK-33 $(n=2)$. B. Effect of RK-33 on undifferentiated and differentiated hESCs determined by MTT assays $(n=2)$. C. Effect of RK-33 on human embryonic fibroblast determined by cell counts $(n=2)$. 
cells (ESCs) compared to their differentiated progeny. ESCs are pluripotent stem cells derived from embryonic blastocyst that like EGCs are unique from ECCs as their proliferation is controlled by cell-to-cell contact and they are genomically stable. In addition to its effect on ECCs and EGCs (data not shown), we found that DDX3 selectively affected proliferation of undifferentiated ESCs, while proliferation of differentiated cells appeared unaltered. This indicates that DDX3 plays a key role in pluripotent stem cell proliferation and its maintenance of the undifferentiated state. Moreover, we found that DDX3 inhibition facilitates differentiation in ECCs and ESCs, and represses pluripotency factors. As a result, inhibition of DDX3 in vivo prevented teratoma formation in NOG mice.

The role in cell cycle regulation and the undifferentiated state of many different types of human pluripotent stem cells indicates that DDX3 has the potential to be a key component of mechanisms that are essential for the maintenance of stem cell renewal and pluripotency. In fact, given the broad functions DDX3 performs across various cell types and with evidence that it effects protein translation in inverse directions depending on the system $(5,26)$, we anticipate that DDX3 probably works in concert with several factors to produce its effects on the stem cell phenotype. For this reason, DDX3 maybe a promising component to target for regenerative medicine. Thus, it would also be of interest in future studies to determine whether DDX3 could also be exploited to generate patient-specific iPSCs for disease modeling, drug testing, or regenerative medicine with patient-derived cells.

\section{MATERIALS AND METHODS}

\section{Ethics statement}

All animal experiments were conducted in accordance with a protocol approved by the Johns Hopkins University Animal Care and Use Committee (Protocol\# MO11M279). All mice were housed in a sterile environment where they had free access to food and water as outlined in our institutional guidelines. Human tissue was obtained as previously described from the University of Washington's NIH-supported tissue repository with Johns Hopkins University Institutional Review Board approval.

\section{Cell culture}

Human ESCs from the H1 line (WiCell, Fed ID\# 0043), human fetal fibroblasts (ATCC, HFF-1) human primordial germ cells (PGCs) and human embryonic germ cells (EGCs) derived from PGCs by the Kerr laboratory as described [41] were cultured on matrigel (BD Biosciences) in $10 \mathrm{~cm}$ cell culture dishes with Dulbecco's modified Eagle's medium/F12-Knockout serum-based media conditioned by mouse embryonic fibroblast cells (MEFs) and supplemented with $4 \mathrm{ng} / \mathrm{mL}$ fibroblast growth factor 2 (FGF2) as described previously [42]. ESCs were routinely maintained on MEFs mitotically inactivated with 5,000 rads of $\gamma$-radiation and passaged every 3-5 days after disaggregation with $1 \mathrm{mg} / \mathrm{ml}$ collagenase in D-PBS. The human embryonal carcinoma line, NTERA-2 cl.D1
hESC

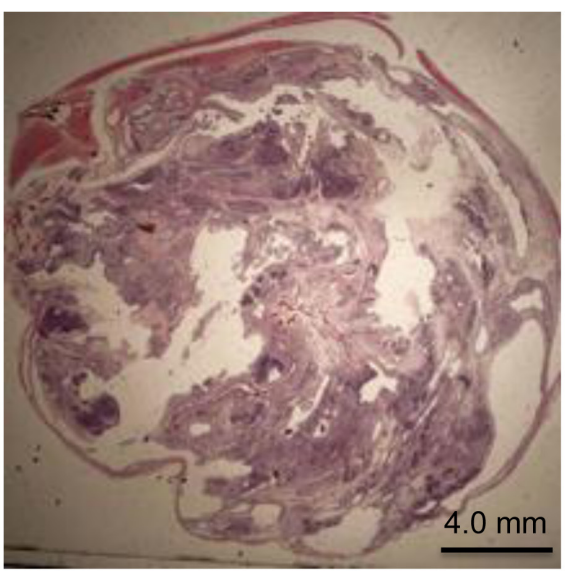

hESC + diff (NP)

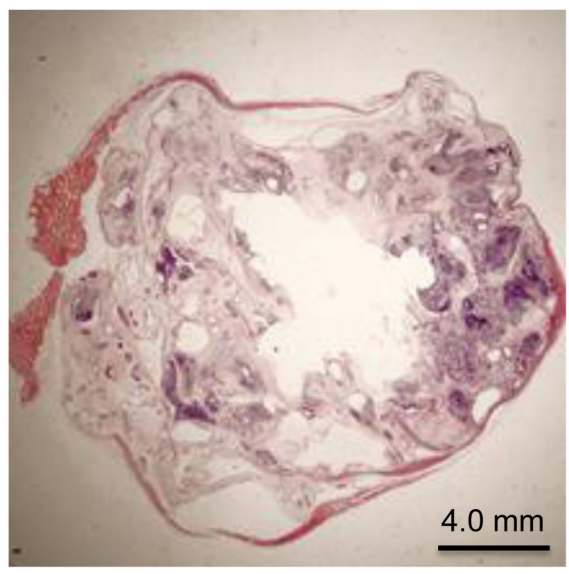

hESC + RK-33

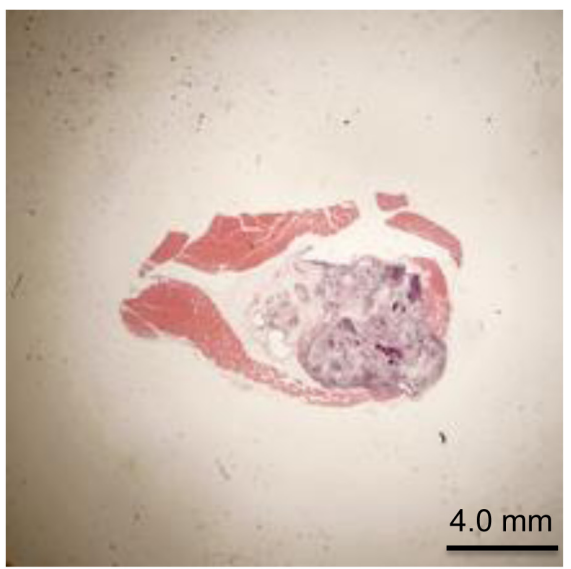

Figure 6: RK-33 enhances differentiation and reduces teratoma formation. Five million of the respective cells in matrigel were injected into the flanks of NOG mice. Following 8 weeks of incubation, tumor volumes were measured and the samples fixed, sectioned and stained with H\&E. The pictures shown represent the actual teratoma volume as related to pixel ratio. The pictures were taken using a $1 \mathrm{X}$ lens. Four animals per group were used for this study. 
(ATCC) was cultured on matrigel-coated plates in 15\% FCS supplemented DMEM media as previously described $[42,43]$.

\section{Immunocytochemistry and quantitative analysis}

For immunocytochemistry, cells were fixed in 4\%PFA for 10 minutes followed by 10 minutes in $0.3 \%$ triton in PBS. All antibodies except DDX3, which was made in our laboratory $[31-33,37,38]$ is commercially available. Briefly, antibodies were diluted in $15 \%$ goat serum in DPBS (1:50 dilution) and incubated with tissue for $1 \mathrm{hr}$ at room temperature. All antibodies were detected using fluorescently labeled secondary antibodies (1:200 dilution; Millipore) in 15\% goat serum in DPBS for $1 \mathrm{hr}$ at room temperature. Cells were counterstained with DAPI (Sigma) to detect nuclei. Negative controls were performed using secondary antibodies alone. For fluorescent quantitative analysis, images were captured using a Nikon E800 microscope and imported to Metamorph Imaging Software (Version 7.7). Total fluorescence units (FU) were calculated by dividing the number of pixels per area (3 areas measured per image), which are in arbitrary units. An adjacent area of the field that was not of interest was also measured to establish background fluorescence as previously described [44] At least, three independent experiments were performed for each treatment from which three separate 48-count tissue culture wells were counted per stain.

\section{MTT cell proliferation assays}

Cells $(5,000)$ were plated onto 96 well plates coated in matrigel with conditioned media obtained from mouse embryonic fibroblasts as described $[45,46]$. The media was replaced daily. MTT assays (Invitrogen) were performed daily for 5 days using $100 \mu \mathrm{l}$ of MTT solution $(5 \mathrm{mg} / \mathrm{ml})$ added to each well and incubated for $3 \mathrm{~h}$ at $37^{\circ} \mathrm{C}$ according to manufacturer's instructions. The formed MTT formazan crystals were dissolved with 500 $\mu \mathrm{L}$ DMSO, and the spectrophotometric assay was carried out at $590 \mathrm{~nm}$ as described. Each condition was done in quadruplicate, and 2 independent experiments were performed.

\section{Teratoma assay}

Five million undifferentiated ESCs were injected subcutaneously into 12 NOD-scidIL-2R $\gamma$ null (NOG) mice as previously described [47]. 24 hours after ESCs transplantation, mice were injected IP with $20 \mathrm{mg} / \mathrm{Kg}$ RK33 or DMSO as control and then repeated injected every other day until sacrifice. After 8 weeks, teratomas were fixed in $4 \%$ PFA, embedded in paraffin at $60^{\circ} \mathrm{C}$ and then cut into 10 micron sections and stained with hematoxylineosin.

\section{Statistical analysis}

Expression levels of DDX3 and the other proteins were compared by chi-square test or t-test as indicated. Logistic regression or ANOVA was used for multivariate analysis. Pearson correlation coefficient was determined for correlation analysis.

All statistical analyses were carried out with SPSS 17.0 for Windows. (SPSS Inc., Chicago, IL, USA), regarding two-sided p-values below 0.05 as significant.

\section{Abbreviations}

ECCs: embryonal carcinoma cells, EGCs: embryonic germ cells, ESCs: embryonic stem cells, FGF2: fibroblast growth factor 2, FU: fluorescence units, hFF: human fetal fibroblasts, MEFs: mouse embryonic fibroblast cells, MTT: tetrazolium MTT (3-(4, 5-dimethylthiazolyl-2)-2,5-diphenyltetrazolium bromide), NOG: NOD/Shi-scid/IL-2R $\gamma^{\text {null }}$, NRN: neural lineage, PGCs: primordial germ cells

\section{Authors' contributions}

$\mathrm{CK}$ and VR conceived and designed the experiments. CK, GB and FV performed the experiments. CK and VR analyzed the data. CK and VR wrote the manuscript. All authors read and approved the manuscript.

\section{CONFLICT OF INTEREST}

Venu Raman has a patent for RK-33 and is on the advisory board of Natsar Pharmaceuticals.

\section{FUNDING}

Research work was support by Flight Attendants Medical Research Institute (FAMRI) to VR.

\section{REFERENCES}

1. Zhou S, Abdouh M, Arena V, Arena M, Arena GO. Reprogramming Malignant Cancer Cells toward a Benign Phenotype following Exposure to Human Embryonic Stem Cell Microenvironment. PLoS One. 2017; 12: e0169899. doi: 10.1371/journal.pone.0169899.

2. Rizzino A, Wuebben EL. Sox2/Oct4: A delicately balanced partnership in pluripotent stem cells and embryogenesis. Biochimica et biophysica acta. 2016; 1859: 780-91. doi: 10.1016/j.bbagrm.2016.03.006. 
3. Hawkins K, Joy S, McKay T. Cell signalling pathways underlying induced pluripotent stem cell reprogramming. World journal of stem cells. 2014; 6: 620-8. doi: 10.4252/ wjsc.v6.i5.620.

4. Heng JC, Orlov YL, Ng HH. Transcription factors for the modulation of pluripotency and reprogramming. Cold Spring Harbor symposia on quantitative biology. 2010; 75 : 237-44. doi: 10.1101/sqb.2010.75.003.

5. Bibikova M, Chudin E, Wu B, Zhou L, Garcia EW, Liu Y, Shin S, Plaia TW, Auerbach JM, Arking DE, Gonzalez R, Crook J, Davidson B, et al. Human embryonic stem cells have a unique epigenetic signature. Genome research. 2006; 16: 1075-83. doi: 10.1101/gr.5319906.

6. Li B, Su T, Ferrari R, Li JY, Kurdistani SK. A unique epigenetic signature is associated with active DNA replication loci in human embryonic stem cells. Epigenetics. 2014; 9: 257-67. doi: 10.4161/epi.26870.

7. Gregory S, Swamy S, Hewitt Z, Wood A, Weightman $\mathrm{R}$, Moore H. Autophagic response to cell culture stress in pluripotent stem cells. Biochemical and biophysical research communications. 2016; 473: 758-63. doi: 10.1016/j.bbrc.2015.09.080.

8. Cho YH, Han KM, Kim D, Lee J, Lee SH, Choi KW, Kim J, Han YM. Autophagy regulates homeostasis of pluripotencyassociated proteins in hESCs. Stem Cells. 2014; 32: 424-35. doi: 10.1002/stem.1589.

9. Tra T, Gong L, Kao LP, Li XL, Grandela C, Devenish RJ, Wolvetang E, Prescott M. Autophagy in human embryonic stem cells. PLoS One. 2011; 6: e27485. doi: 10.1371/ journal.pone.0027485.

10. White J, Dalton S. Cell cycle control of embryonic stem cells. Stem cell reviews. 2005; 1: 131-8. doi: 10.1385/ SCR:1:2:131.

11. El-Badawy A, El-Badri N. The cell cycle as a brake for beta-cell regeneration from embryonic stem cells. Stem cell research \& therapy. 2016; 7: 9. doi: 10.1186/s13287-0150274-z.

12. Barta T, Dolezalova D, Holubcova Z, Hampl A. Cell cycle regulation in human embryonic stem cells: links to adaptation to cell culture. Experimental biology and medicine. 2013; 238: 271-5. doi: 10.1177/1535370213480711.

13. Ariumi Y. Multiple functions of DDX3 RNA helicase in gene regulation, tumorigenesis, and viral infection. Frontiers in genetics. 2014; 5: 423. doi: 10.3389/fgene.2014.00423.

14. Choi YJ, Lee SG. The DEAD-box RNA helicase DDX3 interacts with DDX5, co-localizes with it in the cytoplasm during the G2/M phase of the cycle, and affects its shuttling during mRNP export. Journal of cellular biochemistry. 2012; 113: 985-96. doi: 10.1002/jcb.23428.

15. Gaouar O, Germain H. mRNA export: threading the needle. Frontiers in plant science. 2013; 4: 59. doi: 10.3389/ fpls.2013.00059.
16. Montpetit B, Seeliger MA, Weis K. Analysis of DEAD-box proteins in mRNA export. Methods in enzymology. 2012; 511: 239-54. doi: 10.1016/B978-0-12-396546-2.00011-5.

17. Bol GM, Xie M, Raman V. DDX3, a potential target for cancer treatment. Molecular cancer. 2015; 14: 188. doi: 10.1186/s12943-015-0461-7.

18. Zhao L, Mao Y, Zhou J, Zhao Y, Cao Y, Chen X. Multifunctional DDX3: dual roles in various cancer development and its related signaling pathways. American journal of cancer research. 2016; 6: 387-402.

19. Kim YS, Lee SG, Park SH, Song K. Gene structure of the human DDX3 and chromosome mapping of its related sequences. Molecules and cells. 2001; 12: 209-14.

20. Lahn BT, Page DC. Functional coherence of the human $Y$ chromosome. Science. 1997; 278: 675-80.

21. Kotov AA, Olenkina OM, Godneeva BK, Adashev VE, Olenina LV. Progress in understanding the molecular functions of DDX3Y (DBY) in male germ cell development and maintenance. Bioscience trends. 2017; 11: 46-53. doi: 10.5582/bst.2016.01216.

22. Ramathal C, Angulo B, Sukhwani M, Cui J, DurruthyDurruthy J, Fang F, Schanes P, Turek PJ, Orwig KE, Reijo Pera R. DDX3Y gene rescue of a Y chromosome AZFa deletion restores germ cell formation and transcriptional programs. Scientific reports. 2015; 5: 15041. doi: 10.1038/ srep15041.

23. Wang X, Wang M, Dong W, Li Y, Zheng X, Piao F, $\mathrm{Li}$ S. Subchronic exposure to lead acetate inhibits spermatogenesis and downregulates the expression of Ddx3y in testis of mice. Reproductive toxicology. 2013; 42: 242-50. doi: 10.1016/j.reprotox.2013.10.003.

24. Gueler B, Sonne SB, Zimmer J, Hilscher B, Hilscher W, Graem N, Rajpert-De Meyts E, Vogt PH. AZFa protein DDX3Y is differentially expressed in human male germ cells during development and in testicular tumours: new evidence for phenotypic plasticity of germ cells. Human Reproduction. 2012; 27: 1547-55. doi: 10.1093/humrep/ des047.

25. Lardone MC, Parodi DA, Valdevenito R, Ebensperger M, Piottante A, Madariaga M, Smith R, Pommer R, Zambrano N, Castro A. Quantification of DDX3Y, RBMY1, DAZ and TSPY mRNAs in testes of patients with severe impairment of spermatogenesis. Molecular human reproduction. 2007; 13: 705-12. doi: 10.1093/molehr/gam057.

26. Mazeyrat S, Saut N, Sargent CA, Grimmond S, Longepied G, Ehrmann IE, Ellis PS, Greenfield A, Affara NA, Mitchell MJ. The mouse $\mathrm{Y}$ chromosome interval necessary for spermatogonial proliferation is gene dense with syntenic homology to the human AZFa region. Human molecular genetics. 1998; 7: 1713-24.

27. Solana J, Romero R. SpolvlgA is a DDX3/PL10-related DEAD-box RNA helicase expressed in blastomeres and embryonic cells in planarian embryonic development. 
International journal of biological sciences. 2009; 5: 64-73.

28. Rosner A, Paz G, Rinkevich B. Divergent roles of the DEAD-box protein BS-PL10, the urochordate homologue of human DDX3 and DDX3Y proteins, in colony astogeny and ontogeny. Developmental dynamics : an official publication of the American Association of Anatomists. 2006; 235: 1508-21. doi: 10.1002/dvdy.20728.

29. Chen CY, Chan CH, Chen CM, Tsai YS, Tsai TY, Wu Lee YH, You LR. Targeted inactivation of murine Ddx3x: essential roles of Ddx3x in placentation and embryogenesis. Human molecular genetics. 2016; 25: 2905-22. doi: 10.1093/hmg/ddw143.

30. Chang TC, Liu WS. The molecular evolution of PL10 homologs. BMC Evol Biol. 2010; 10: 127. doi: 10.1186/1471-2148-10-127.

31. Bol GM, Vesuna F, Xie M, Zeng J, Aziz K, Gandhi N, Levine A, Irving A, Korz D, Tantravedi S, Heerma van Voss MR, Gabrielson K, Bordt EA, et al. Targeting DDX3 with a small molecule inhibitor for lung cancer therapy. EMBO Mol Med. 2015; 7: 648-69. doi: 10.15252/ emmm.201404368.

32. Heerma van Voss MR, Vesuna F, Trumpi K, Brilliant J, Berlinicke C, de Leng W, Kranenburg O, Offerhaus GJ, Burger H, van der Wall E, van Diest PJ, Raman V. Identification of the DEAD box RNA helicase DDX3 as a therapeutic target in colorectal cancer. Oncotarget. 2015; 6 : 28312-26. doi: 10.18632/oncotarget.4873.

33. Xie M, Vesuna F, Tantravedi S, Bol GM, Heerma van Voss MR, Nugent K, Malek R, Gabrielson K, van Diest PJ, Tran PT, Raman V. RK-33 Radiosensitizes Prostate Cancer Cells by Blocking the RNA Helicase DDX3. Cancer Research. 2016; 76: 6340-50. doi: 10.1158/0008-5472.CAN-16-0440.

34. Kerr CL, Hill, C.M., Blumenthal, P, and Gearhart, J.D. Expression of Pluripotent Stem Cell Markers in the Human Fetal Ovary. Human Reproduction. 2008.

35. Kerr CL, Gearhart JD, Elliott AM, Donovan PJ. Embryonic germ cells: when germ cells become stem cells. Semin Reprod Med. 2006; 24: 304-13.

36. Kerr DA, Llado J, Shamblott MJ, Maragakis NJ, Irani DN, Crawford TO, Krishnan C, Dike S, Gearhart JD, Rothstein JD. Human embryonic germ cell derivatives facilitate motor recovery of rats with diffuse motor neuron injury. J Neurosci. 2003; 23: 5131-40.

37. Botlagunta M, Vesuna F, Mironchik Y, Raman A, Lisok A, Winnard P, Jr., Mukadam S, Van Diest P, Chen JH, Farabaugh P, Patel AH, Raman V. Oncogenic role of DDX3 in breast cancer biogenesis. Oncogene. 2008; 27: 3912-22. doi: 10.1038/onc.2008.33.
38. Heerma van Voss MR, Schrijver WA, Ter Hoeve ND, Hoefnagel LD, Manson QF, van der Wall E, Raman V, van Diest PJ, Dutch Distant Breast Cancer Metastases C. The prognostic effect of DDX3 upregulation in distant breast cancer metastases. Clin Exp Metastasis. 2017; 34: 85-92. doi: 10.1007/s10585-016-9832-8.

39. Heerma van Voss MR, van Kempen PM, Noorlag R, van Diest PJ, Willems SM, Raman V. DDX3 has divergent roles in head and neck squamous cell carcinomas in smoking versus non-smoking patients. Oral Dis. 2015; 21: 270-1. doi: 10.1111/odi.12299.

40. Bol GM, Raman V, van der Groep P, Vermeulen JF, Patel AH, van der Wall E, van Diest PJ. Expression of the RNA helicase DDX3 and the hypoxia response in breast cancer. PLoS One. 2013; 8: e63548.

41. Bazley FA, Liu CF, Yuan X, Hao H, All AH, De Los Angeles A, Zambidis ET, Gearhart JD, Kerr CL. Direct Reprogramming of Human Primordial Germ Cells into Induced Pluripotent Stem Cells: Efficient Generation of Genetically Engineered Germ Cells. Stem cells and development. 2015; 24: 2634-48. doi: 10.1089/ scd.2015.0100.

42. Chaerkady R, Kerr CL, Kandasamy K, Marimuthu A, Gearhart JD, Pandey A. Comparative proteomics of human embryonic stem cells and embryonal carcinoma cells. Proteomics.

43. Andrews PW, Damjanov I, Simon D, Banting GS, Carlin C, Dracopoli NC, Fogh J. Pluripotent embryonal carcinoma clones derived from the human teratocarcinoma cell line Tera-2. Differentiation in vivo and in vitro. Lab Invest. 1984; 50: 147-62.

44. Kerr CL, Shamblott MJ, Gearhart JD. Pluripotent stem cells from germ cells. Methods Enzymol. 2006; 419: 400-26.

45. Swelstad BB, Kerr CL. Current protocols in the generation of pluripotent stem cells: theoretical, methodological and clinical considerations. Stem cells and cloning : advances and applications. 2009; 3: 13-27.

46. All AH, Bazley FA, Gupta S, Pashai N, Hu C, Pourmorteza A, Kerr C. Human embryonic stem cell-derived oligodendrocyte progenitors aid in functional recovery of sensory pathways following contusive spinal cord injury. PLoS One. 2012; 7: e47645. doi: 10.1371/journal. pone. 0047645 .

47. Chen G, Ye Z, Yu X, Zou J, Mali P, Brodsky RA, Cheng L. Trophoblast differentiation defect in human embryonic stem cells lacking PIG-A and GPI-anchored cell-surface proteins. Cell Stem Cell. 2008; 2: 345-55. 